\title{
Akivis superalgebras and speciality
}

\author{
Helena Albuquerque * Ana Paula Santana $^{\dagger}$
}

October 31, 2018

\begin{abstract}
In honour of Ivan Shestakov on the occasion of his 60th birthday
\end{abstract}
\begin{abstract}
In this paper we define Akivis superalgebra and study enveloping superalgebras for this class of algebras, proving an analogous of the PBW Theorem.

Lie and Malcev superalgebras are examples of Akivis superalgebras. For these particular superalgebras, we describe the connection between the classical enveloping superalgebras and the corresponding generalized concept defined in this work.
\end{abstract}

Keywords: Lie superalgebras; Malcev superalgebras; Enveloping superalgebras

AMS-Classification: 15A63, 17A70

\section{Introduction}

Definition 1. The supervector space $M=M_{0} \oplus M_{1}$ is called an Akivis superalgebra if it is endowed with two operations:

- a bilinear superanticommutative map [,] that induces on $M$ a structure of superalgebra;

- a trilinear map A, compatible with the gradation (i.e, $A\left(M_{\alpha}, M_{\beta}, M_{\gamma}\right) \subseteq M_{\alpha+\beta+\gamma}$, all $\left.\alpha, \beta, \gamma \in Z_{2}\right)$, satisfying the following identity:

$[[x, y], z]+(-1)^{\alpha(\beta+\gamma)}[[y, z], x]+(-1)^{\gamma(\beta+\alpha)}[[z, x], y]=A(x, y, z)+(-1)^{\alpha(\beta+\gamma)} A(y, z, x)+$ $+(-1)^{\gamma(\beta+\alpha)} A(z, x, y)-(-1)^{\alpha \beta} A(y, x, z)-(-1)^{\alpha(\beta+\gamma)+\beta \gamma} A(z, y, x)-(-1)^{\gamma \beta} A(x, z, y)$, for homogeneous elements $x \in M_{\alpha}, y \in M_{\beta}, z \in M_{\gamma}$, all $\alpha, \beta, \gamma \in Z_{2}$.

This superalgebra will be denoted in this work by $(M,[], A$,$) , or simply M$, if no confusion arises.

This definition is a generalization of the notion of Akivis algebra presented by I. Shestakov in [8]. In fact, the even part of an Akivis superalgebra is an Akivis algebra. Akivis algebras were introduced by M. A. Akivis in [1] as local algebras of local analytic loops.

* Departamento de Matemática, Faculdade de Ciências e Tecnologia da Universidade de Coimbra, Apartado 3008, 3001-454 Coimbra, Portugal; E-mail: lena@mat.uc.pt. Financial Support by CMUC-FCT gratefully acknowledged

${ }^{\dagger}$ Departamento de Matemática, Faculdade de Ciências e Tecnologia da Universidade de Coimbra, Apartado 3008, 3001-454 Coimbra, Portugal; E-mail: aps@mat.uc.pt. Financial Support by CMUC-FCT gratefully acknowledged 
In this paper, we consider Akivis superalgebras over a field $K$ of characteristic different from 2 and 3. It is our aim to study the enveloping superalgebra of an Akivis superalgebra and to prove an analogous of the PBW Theorem. Our approach is similar to the one used in [8] but, as it is expected, the superization of the results imply more elaborated calculations and arguments. This is particularly evident in the definition of the superalgebra $\tilde{V}(M)$ studied in Section 5.

Given a superalgebra $W$ with multiplication $($,$) , we will denote by W^{-}$the superalgebra with underlying supervector space $W$ and multiplication [,] given by $[x, y]=(x, y)-(-1)^{\alpha \beta}(y, x)$ for homogeneous elements $x \in W_{\alpha}, y \in W_{\beta}$ (and extended by linearity to every element of $W$ ). It is known that if $W$ is an associative superalgebra then the superalgebra $W^{-}$is a Lie superalgebra, and if $W$ is an alternative superalgebra then $W^{-}$is a Malcev superalgebra. Standard calculations show that if $W$ is any superalgebra, the superalgebra $W^{-}$is an Akivis superalgebra for the trilinear map $A(x, y, z)=(x y) z-x(y z)$. This superalgebra will be denoted in this work by $W^{A}$.

We recall that a superalgebra $S$ is said to be special if it is isomorphic to $U^{-}$for some superalgebra $U$.

It is well known that every Lie superalgebra is isomorphic to a superalgebra $S^{-}$, where $S$ is an associative superalgebra. In fact, for any Lie superalgebra $L$, let $T(L)$ denote the associative tensor superalgebra of the vector space $L$, and consider its bilateral ideal $I$ generated by the homogeneous elements $x \otimes y-(-1)^{\alpha \beta} y \otimes x-[x, y]$, all $x \in M_{\alpha}, y \in M_{\beta}, \alpha, \beta \in Z_{2}$. Then the associative superalgebra $T(L) / I$ is the universal enveloping superalgebra of $L$ and $L$ is isomorphic to a subsuperalgebra of $(T(L) / I)^{-}$.

It is an open problem to know if a Malcev superalgebra is isomorphic to $S^{-}$for some alternative superalgebra $S$. This problem was solved only partially in [3] and [4]. There it was shown that, in some cases, a Malcev algebra is isomorphic to a subalgebra of $\operatorname{Nat}(T)^{-}$for an algebra $T$, where $\operatorname{Nat}(T)$ denotes the generalized alternative nucleous of $T$.

In this work, we prove that an Akivis superalgebra $M$ defined over a field $K$ of characteristic different from 2 and 3 is isomorphically embedded in $\tilde{U}(M)^{A}$, where $\tilde{U}(M)$ is its enveloping superalgebra. So $M$ is special.

\section{Examples of Akivis superalgebras}

Lie superalgebras and more generally Malcev superalgebras are Akivis superalgebras. For the first class, we consider the trilinear map A to be the zero map and, for the second class, we take $A(x, y, z)=1 / 6 S J(x, y, z)$. Here $S J(x, y, z)$ denotes the superjacobian

$$
S J(x, y, z)=[[x, y], z]+(-1)^{\alpha(\beta+\gamma)}[[y, z], x]+(-1)^{\gamma(\beta+\alpha)}[[z, x], y],
$$

of the homogeneous elements $x \in M_{\alpha}, y \in M_{\beta}, z \in M_{\gamma},\left(\alpha, \beta, \gamma \in Z_{2}\right)$.

Next, we give two examples of Akivis superalgebras which are not included in these classes. Consider the algebra of octonions $O$ as the algebra obtained by Cayley-Dickson Process from the quaternions $Q$, with the $Z_{2}$ gradation

$$
O_{0}=Q=<1, e_{1}, e_{2}, e_{3}>\quad \text { and } O_{1}=e_{4} Q=<e_{4}, e_{5}, e_{6}, e_{7}>\text {. }
$$


The multiplication table of the Akivis superalgebra $O^{A}$ is shown below. Note that the even part of this superalgebra is the simple Lie algebra $\operatorname{sl}(2, K)=<e_{1}, e_{2}, e_{3}>$ together with $1 \in Z(O)$.

\begin{tabular}{|c||c|c|c|c|c|c|c|c|}
\hline & 1 & $e_{1}$ & $e_{2}$ & $e_{3}$ & $e_{4}$ & $e_{5}$ & $e_{6}$ & $e_{7}$ \\
\hline \hline 1 & 0 & 0 & 0 & 0 & 0 & 0 & 0 & 0 \\
$e_{1}$ & 0 & 0 & $-2 e_{3}$ & $2 e_{2}$ & $-2 e_{5}$ & $2 e_{4}$ & $2 e_{7}$ & $-2 e_{6}$ \\
$e_{2}$ & 0 & $2 e_{3}$ & 0 & $-2 e_{1}$ & $-2 e_{6}$ & $-2 e_{7}$ & $2 e_{4}$ & $2 e_{5}$ \\
$e_{3}$ & 0 & $-2 e_{2}$ & $2 e_{1}$ & 0 & $-2 e_{7}$ & $2 e_{6}$ & $-2 e_{5}$ & $2 e_{4}$ \\
$e_{4}$ & 0 & $2 e_{5}$ & $2 e_{6}$ & $2 e_{7}$ & -2 & 0 & 0 & 0 \\
$e_{5}$ & 0 & $-2 e_{4}$ & $2 e_{7}$ & $-2 e_{6}$ & 0 & -2 & 0 & 0 \\
$e_{6}$ & 0 & $-2 e_{7}$ & $-2 e_{4}$ & $2 e_{5}$ & 0 & 0 & -2 & 0 \\
$e_{7}$ & 0 & $2 e_{6}$ & $-2 e_{5}$ & $-2 e_{4}$ & 0 & 0 & 0 & -2 \\
\hline
\end{tabular}

This superalgebra is an Akivis superalgebra that is neither a Lie nor a Malcev superalgebra. Indeed, we have that $S J\left(e_{3}, e_{7}, e_{2}\right) \neq 0$ and $\left(\left(e_{4} e_{2}\right) e_{3}\right) e_{5}-\left(\left(e_{2} e_{3}\right) e_{5}\right) e_{4} \neq\left(e_{4} e_{3}\right)\left(e_{2} e_{5}\right)$.

The second class of examples can be obtained using antiassociative superalgebras (nonassociative $Z_{2}$-graded quasialgebras). Consider $D$ a division algebra and $n, m$ natural numbers. In [3] the authors studied the superalgebra $\widetilde{M a t}_{n, m}(D)$ of the $(n+m) \times(n+m)$ matrices over $D$, with the chess-board $Z_{2}$-grading

$$
\begin{array}{r}
\widetilde{M a t}_{n, m}(D)_{0}=\left\{\left(\begin{array}{cc}
a & 0 \\
0 & b
\end{array}\right): a \in \operatorname{Mat}_{n}(D), b \in \operatorname{Mat}_{m}(D)\right\}, \\
\widetilde{M a t}_{n, m}(D)_{1}=\left\{\left(\begin{array}{cc}
0 & v \\
w & 0
\end{array}\right): v \in \operatorname{Mat}_{n \times m}(D), w \in \operatorname{Mat}_{m \times n}(D)\right\}
\end{array}
$$

and with multiplication given by

$$
\left(\begin{array}{ll}
a_{1} & v_{1} \\
w_{1} & b_{1}
\end{array}\right) \cdot\left(\begin{array}{cc}
a_{2} & v_{2} \\
w_{2} & b_{2}
\end{array}\right)=\left(\begin{array}{cc}
a_{1} a_{2}+v_{1} w_{2} & a_{1} v_{2}+v_{1} b_{2} \\
w_{1} a_{2}+b_{1} w_{2} & -w_{1} v_{2}+b_{1} b_{2}
\end{array}\right)
$$

This superalgebra is antiassociative, with even part isomorphic to $\operatorname{Mat}_{n}(D) \times \operatorname{Mat}_{m}(D)$. $\widetilde{M a t}_{n, m}(D)^{A}$ is an Akivis superalgebra that is neither a Lie nor a Malcev superalgebra. In fact, if $E_{i, j}$ denotes the $(n+m) \times(n+m)$ matrix with $(i j)$ entry equal to 1 and all the other entries equal to 0 , then $S J\left(E_{1, n+1}, E_{n+1,1}, E_{1, n+1}\right) \neq 0$ and $2\left(\left(E_{1, n+1} E_{n+1,1}\right) E_{1, n+1}\right) E_{n+1,1}-$ $-\left(\left(E_{n+1,1} E_{1, n+1}\right) E_{n+1,1}\right) E_{1, n+1} \neq E_{1, n+1}^{2} E_{n+1,1}^{2}$.

In the case $m=n=1, \tilde{M} a t_{1,1}(D)^{A}$ has abelian even part and multiplication given by the following table, where $a=E_{11}, b=E_{11}-E_{22}, x=E_{12}, y=E_{21}$ :

\begin{tabular}{|c||c|c|c|c|}
\hline & $\mathrm{a}$ & $b$ & $x$ & $y$ \\
\hline \hline$a$ & 0 & 0 & $\mathrm{x}$ & $-y$ \\
$\mathrm{~b}$ & 0 & 0 & $2 \mathrm{x}$ & $-2 \mathrm{y}$ \\
$x$ & $-\mathrm{x}$ & $-2 \mathrm{x}$ & 0 & $b$ \\
$y$ & $\mathrm{y}$ & $2 \mathrm{y}$ & $b$ & 0 \\
\hline
\end{tabular}




\section{Enveloping superalgebra of an Akivis superalgebra}

In this section we construct and study the universal enveloping superalgebra of an Akivis superalgebra.

Given the Akivis superalgebras $(M,[], A$,$) and \left(N,[,]^{\prime}, A^{\prime}\right)$, by an Akivis homomorphism we mean a superalgebra homomorphism of degree $0, f: M \rightarrow N$, such that, for all $x, y, z \in$ $M, f(A(x, y, z))=A^{\prime}(f(x), f(y), f(z))$.

Definition 2. Let $M$ be an Akivis superalgebra. A pair $(\tilde{U}, \iota)$ is an universal enveloping superalgebra of $M$ if $\tilde{U}$ is a superalgebra and $\iota: M \rightarrow \tilde{U}^{A}$ is an Akivis homomorphism satisfying the following condition: given any superalgebra $W$ and any Akivis homomorphism $\theta: M \rightarrow W^{A}$, there is a unique superalgebra homomorphism of degree $0, \tilde{\theta}: \tilde{U} \rightarrow W$, such that $\theta=\tilde{\theta} \iota$.

In a similar way to the one used in the classical case for Lie superalgebras we can prove the following:

Proposition 1. 1) The universal enveloping superalgebra of an Akivis superalgebra is unique up to isomorphism;

2) Let $M$ be an Akivis superalgebra and $(\tilde{U}, \iota)$ its universal enveloping superalgebra. Then the superalgebra $\tilde{U}$ is generated by $\iota(M)$ and $K$;

3)Consider two Akivis superalgebras $M_{1}$ and $M_{2}$ with universal enveloping superalgebras $\left(\tilde{U}_{1}, \iota_{1}\right)$ and $\left(\tilde{U}_{2}, \iota_{2}\right)$, respectively. If there is an Akivis homomorphism $\phi: M_{1} \rightarrow M_{2}$ then there is a superalgebra homomorphism $\tilde{\phi}: \tilde{U}_{1} \rightarrow \tilde{U}_{2}$ such that $\tilde{\phi} \iota_{1}=\iota_{2} \phi$.

4)Let $M$ be an Akivis superalgebra with universal enveloping superalgebra $(\tilde{U}, \iota)$. Let I be a graded ideal in $M$ and let $J$ be the graded ideal of $\tilde{U}$ generated by $\iota(I)$. If $m \in M$, the map $\lambda: m+I \rightarrow \iota(m)+J$ is an Akivis homomorphism of $M / I$ in $(\tilde{U} / J)^{A}$ and $(\tilde{U} / J, \lambda)$ is the universal enveloping superalgebra of $M / I$.

Next we will construct the universal enveloping superalgebra of the Akivis superalgebra $(M,[], A$,$) . We start by considering the nonassociative Z$-graded tensor algebra of $M$

$$
\tilde{T}(M)=\oplus_{n \in Z} \tilde{T}^{n}(M),
$$

where $\tilde{T}^{n}(M)=0$, if $n<0, \tilde{T}^{0}(M)=K, \tilde{T}^{1}(M)=M$ and $\tilde{T}^{n}(M)=\oplus_{i=1}^{n-1} \tilde{T}^{i}(M) \otimes \tilde{T}^{n-i}(M)$, for $n \geq 2$, with multiplication defined by $x y=x \otimes y$. $\tilde{T}(M)$ is a superalgebra with the $Z_{2^{-}}$ gradation

$$
\tilde{T}(M)=\left(\oplus_{n \geq 0} \tilde{T}^{n}(M)_{0}\right) \oplus\left(\oplus_{n \geq 1} \tilde{T}^{n}(M)_{1}\right),
$$

where

$$
\tilde{T}^{n}(M)_{\gamma}=\oplus_{i=1}^{n-1} \oplus_{\alpha+\beta=\gamma}\left(\tilde{T}^{i}(M)_{\alpha} \otimes \tilde{T}^{n-i}(M)_{\beta}\right), \gamma \in Z_{2} .
$$

Let $I$ be the $Z_{2}$-graded ideal of $\tilde{T}(M)$ generated by the homogeneous elements

$$
x \otimes y-(-1)^{\alpha \beta} y \otimes x-[x, y] \text { and }(x \otimes y) \otimes z-x \otimes(y \otimes z)-A(x, y, z),
$$

for $x \in M_{\alpha}, y \in M_{\beta}, z \in M_{\gamma}$. The quocient algebra $\tilde{U}(M)=\tilde{T}(M) / I$ is a superalgebra with the natural $Z_{2}$-gradation induced by the graded ideal $I$. Consider the map $\iota: M \rightarrow \tilde{T}(M) \rightarrow$ $\tilde{U}(M)$ obtained by the composition of the canonical injection with the quocient map. It is obvious that $\iota$ is an Akivis homomorphism between $M$ and $\tilde{U}(M)^{A}$. 
Proposition 2. The superalgebra $(\tilde{T}(M) / I, \iota)$ is the universal enveloping superalgebra of the Akivis superalgebra $M$.

Proof: Given any superalgebra $W$ and an Akivis homomorphism $f: M \rightarrow W^{A}$, we need to prove that there is a unique homomorphism $\tilde{f}: \tilde{U}(M) \rightarrow W$ such that $f=\tilde{f} \iota$.

Using the universal property of tensor products it is easy to see that there is a unique superalgebra homomorphism of degree $0, f^{*}: \tilde{T}(M) \rightarrow W$, such that $f^{*}(m)=f(m)$, for all $m \in M$. The fact that $f$ is an Akivis homomorphism implies that $I \subseteq$ ker $f^{*}$. Hence, there is an homomorphism of superalgebras of degree $0, \tilde{f}: \tilde{U}(M) \rightarrow W$ such that $\tilde{f}(\iota(m))=f^{*}(m)=f(m)$, all $m \in M$. As $\tilde{U}(M)$ is generated by $\mathrm{K}$ and $\iota(M)$, the unicity of $\tilde{f}$ follows.

\section{Enveloping superalgebras of Lie and Malcev superalgebras}

In [4], Pérez-Izquierdo studied enveloping algebras of Sabinin algebras, showing that these generalize the classical notions of enveloping algebras for the particular cases of Lie algebras and Malcev algebras. In this section, we will study the connections between the classical definitions and the definition of enveloping superalgebras of Lie and Malcev superalgebras considered as Akivis superalgebras.

Given a Lie superalgebra $L$, denote by $(\tilde{U}(L), \iota)$ its enveloping superalgebra as an Akivis superalgebra and by $(U(L), \sigma)$ its classical universal enveloping superalgebra. Clearly $\sigma$ is an Akivis homomorphism from $L$ to $U(L)^{A}$. So there is a unique homomorphism of superalgebras $\tilde{\sigma}$ such that $\tilde{\sigma} \iota=\sigma$. As $U(L)$ is generated by $\mathrm{K}$ and $\sigma(L), \tilde{\sigma}$ is surjective. So $U(L)$ is an epimorphic image of $\tilde{U}(L)$ by $\tilde{\sigma}$, i. e.,

$$
U(L) \simeq \tilde{U}(L) / \operatorname{ker} \tilde{\sigma} .
$$

Suppose now that $N$ is a Malcev superalgebra. Superizing the theory exposed in [5], we can naturally define the classical enveloping superalgebra of $N$ as the superalgebra $(\tilde{T}(N) / \tilde{I}, \tilde{\iota})$, where $\tilde{I}$ is the graded ideal of $\tilde{T}(N)$ generated by the homogeneous elements

$$
a \otimes b-(-1)^{\alpha \beta} b \otimes a-[a, b], \quad(a, x, y)+(-1)^{\alpha \gamma}(x, a, y), \quad(x, a, y)+(-1)^{\alpha \xi}(x, y, a),
$$

all $a \in N_{\alpha}, b \in N_{\beta}, x \in \tilde{T}(N)_{\gamma}, y \in \tilde{T}(N)_{\xi}$, and where $(x, y, z)=(x y) z-x(y z)$ denotes the usual associator. The map $\tilde{\iota}$ is the composition of the canonical injection from $N$ to $\tilde{T}(N)$ with the canonical epimorphism $\mu: \tilde{T}(N) \rightarrow \tilde{T}(N) / \tilde{I}$.

We will prove that $\tilde{T}(N) / \tilde{I}$ is an epimorphic image of $\tilde{U}(N)$. For this, we show that $I \subseteq \tilde{I}$ and so $\mu$ gives rise to the epimorphism $\tilde{\mu}: \tilde{T}(N) / I \rightarrow \tilde{T}(N) / \tilde{I}$ defined by $\tilde{\mu}(n+I)=n+\tilde{I}$, all $n \in \tilde{T}(N)$. To see that $I \subseteq \tilde{I}$ notice that, for all homogeneous elements $x \in N_{\gamma}, y \in N_{\xi}, z \in$ $N_{\alpha}$, we have that

$S J(x, y, z)-3(x y) z=(-1)^{\alpha(\gamma+\xi)}\left[(z, x, y)+(-1)^{\alpha \gamma}(x, z, y)\right]-(-1)^{\alpha \xi}\left[(x, z, y)+(-1)^{\alpha \xi}(x, y, z)\right]$

is an element of $\tilde{I}$ and so $S J(x, y, z)-3(x y) z+S J(y, z, x)-3(y z) x \in \tilde{I}$. Therefore, $S J(x, y, z) \in \tilde{I}$ and $(x, y, z) \in \tilde{I}$. As the generators of $I$ are in $\tilde{I}$, we can conclude that $I \subseteq \tilde{I}$. 


\section{$5 \quad$ Speciality of Akivis superalgebras}

The canonical filtration of $\tilde{T}(M), \tilde{T}_{0}(M) \subseteq \tilde{T}_{1}(M) \subseteq \ldots$, where $\tilde{T}_{0}(M)=K$, and $\tilde{T}_{n}(M)=$ $\oplus_{i=0}^{n} \tilde{T}^{i}(M), n>0$, gives rise to the canonical filtration of $\tilde{U}(M), \tilde{U}_{0}(M) \subseteq \tilde{U}_{1}(M) \subseteq \ldots$, where $\tilde{U}_{n}(M)=\tilde{T}_{n}(M)+I$.

Associated with this filtration there is the $Z$-graded superalgebra,

$$
\operatorname{gr} \tilde{U}(M)=\oplus_{n \in Z}(\operatorname{gr} \tilde{U}(M))_{n},
$$

where $(\operatorname{gr} \tilde{U}(M))_{n}=0$, if $n<0,(\operatorname{gr} \tilde{U}(M))_{0}=K,(\operatorname{gr} \tilde{U}(M))_{n}=\tilde{U}_{n}(M) / \tilde{U}_{n-1}(M)$, for $n \geq 1$, with multiplication given by

$$
\left(a+\tilde{U}_{i-1}(M)\right)\left(b+\tilde{U}_{j-1}(M)\right)=a b+\tilde{U}_{i+j-1}(M)
$$

for $a \in \tilde{U}_{i}(M)$ and $b \in \tilde{U}_{j}(M)$. For simplicity we identify $(\operatorname{gr} \tilde{U}(M))_{1}$ with $\iota(M)$.

Now consider the classical tensor algebra $T(M)$ of $M$, that is naturally a $Z$-graded associative superalgebra,

$$
T(M)=\oplus_{n \in Z} T^{n}(M)
$$

where $T^{n}(M)=0$ if $n<0, T^{0}(M)=K, T^{n}(M)=M \otimes M \otimes \ldots \otimes M$ ( $n$ times) if $n>0$. Let $J$ be the ideal of $T(M)$ generated by the homogeneous elements $x \otimes y-(-1)^{\alpha \beta} y \otimes x$, all $x \in$ $M_{\alpha}, y \in M_{\beta}, \alpha, \beta \in Z_{2}$. The associative Z-graded quotient superalgebra $S(M)=T(M) / J$ is called the supersymmetric superalgebra of $M$. Note that as associative Z-graded algebra the homogeneous spaces of $S(M)$ are $S^{n}(M)=T^{n}(M)+J$ and as a superalgebra we have $S(M)_{\alpha}=\oplus_{n \in Z}\left(\oplus_{\alpha_{1}+\ldots+\alpha_{n}=\alpha}\left(M_{\alpha_{1}} \otimes \ldots \otimes M_{\alpha_{n}}+J\right)\right)$. Since the generators of $J$ lie in $T^{2}(M)$, we identify $S^{0}(M)$ with $K$ and $S^{1}(M)$ with $M$.

We will now construct from $S(M)$ a nonassociative superalgebra $V(M)$ which will play in this work the role that the symmetric algebra plays in the classical case of the PBW Theorem.

We define the $Z$-graded supervector space $V(M)=\oplus_{n \in Z} V^{n}(M)$, where the subspaces $V^{n}(M)$ are defined by

$$
V^{n}(M)=S^{n}(M), \text { if } n \leq 3, \text { and } V^{n}(M)=\oplus_{i=1}^{n-1}\left(V^{i}(M) \otimes V^{n-i}(M)\right) \text {, if } n>3 .
$$

We turn $V(M)$ into a superalgebra by defining the multiplication for homogeneous elements $v_{i} \in V^{i}(M), v_{j} \in V^{j}(M)$ by $v_{i} \cdot v_{j}=v_{i} v_{j}$ if $i+j \leq 3$ and $v_{i} \cdot v_{j}=v_{i} \otimes v_{j}$ if $i+j>3$ (where juxtaposition of elements means the product of these elements in $S(M)$ ).

Lemma 1. The superalgebra $V(M)$ is the enveloping Akivis superalgebra of the trivial Akivis superalgebra with underlying vector space $M$, i.e., $V(M) \cong \tilde{T}(M) / I^{*}$ where $I^{*}$ is the ideal of $\tilde{T}(M)$ generated by the homogeneous elements $x \otimes y-(-1)^{\alpha \beta} y \otimes x,(x \otimes y) \otimes z-x \otimes(y \otimes z)$, all $x \in M_{\alpha}, y \in M_{\beta}, z \in M_{\gamma}$.

Proof: The inclusion map $\pi: M \rightarrow V(M)^{A}$ is an Akivis homomorphism. Therefore, as $\tilde{T}(M) / I^{*}$ is the enveloping superalgebra of the trivial Akivis superalgebra obtained from $M$, there is a superalgebra map of degree $0, \tilde{\pi}: \tilde{T}(M) / I^{*} \rightarrow V(M)$ such that $\tilde{\pi}\left(m+I^{*}\right)=m$, all $m \in M$.

Notice that in $\tilde{T}(M) / I^{*}$ we have $x \otimes y-(-1)^{\alpha \beta} y \otimes x+I^{*}=0$ and $(x \otimes y) \otimes z-x \otimes(y \otimes z)+I^{*}=0$, for homogeneous elements $x, y, z$. Hence, using the universal property of the tensor products, we may define linear maps $\rho_{1}: M \rightarrow \tilde{T}(M) / I^{*}, \rho_{2}: M \otimes M \rightarrow \tilde{T}(M) / I^{*}, \rho_{3}: M \otimes M \otimes M \rightarrow$ $\tilde{T}(M) / I^{*}$ by $\rho_{i}(a)=a+I^{*}, i=1,2,3$. Clearly, $J \cap M \otimes M \subseteq \operatorname{ker} \rho_{2}$ and $J \cap M \otimes M \otimes M \subseteq \operatorname{ker} \rho_{3}$. 
Therefore, there are linear maps $\tilde{\rho}_{i}: V^{i}(M) \rightarrow \tilde{T}(M) / I^{*}$ defined by $\tilde{\rho}_{i}(a+J)=a+I^{*}, \mathrm{i}=1$, 2 , 3. Using once more the universal property of tensor products and induction, one can extend these maps to an homomorphism of superalgebras of degree $0, \tilde{\rho}: V(M) \rightarrow \tilde{T}(M) / I^{*}$ satisfying $\tilde{\rho}(m)=m+I^{*}$ for all $m \in M$. The maps $\tilde{\rho}$ and $\tilde{\pi}$ are inverse of each other. So $\tilde{\pi}$ is an isomorphism.

Lemma 2. There exists an epimorphism of Z-graded superalgebras $\tilde{\tau}: \tilde{T}(M) / I^{*} \rightarrow \operatorname{gr} \tilde{U}(M)$, of degree 0 , such that $\tilde{\tau}\left(m+I^{*}\right)=\iota(m)$, all $m \in M$.

Proof: Consider the natural epimorphism of $Z$-graded algebras $\tau: \tilde{T}(M) \rightarrow \operatorname{gr} \tilde{U}(M)$ given by $\tau(a)=(a+I)+\tilde{U}_{n-1}(M)$, for each $a \in \tilde{T}^{n}(M)$. Notice that, since we identify $(\operatorname{gr} \tilde{U}(M))_{1}$ with $\iota(M)=M+I$, then $\tau(m)=\iota(m)$, for all $m \in M$. To see that $\tau$ preserves the gradation, recall that for $\alpha \in Z_{2}, \tilde{T}(M)_{\alpha}=\oplus_{n \geq 0} \tilde{T}^{n}(M)_{\alpha}$ and

$$
(g r \tilde{U}(M))_{\alpha}=\oplus_{n \geq 0}\left(\tilde{U}_{n}(M) / \tilde{U}_{n-1}(M)\right)_{\alpha}=\oplus_{n \geq 0}\left(\oplus_{i=0}^{n}\left(\tilde{T}^{i}(M)_{\alpha}+I\right)+\tilde{U}_{n-1}(M)\right) .
$$

So if $a \in \tilde{T}^{n}(M)_{\alpha}$, then $\tau(a)=(a+I)+\tilde{U}_{n-1}(M) \in\left(\tilde{T}^{n}(M)_{\alpha}+I\right)+\tilde{U}_{n-1}(M)$. Hence

$$
\left.\tau\left(\tilde{T}(M)_{\alpha}\right)=\sum_{n \geq 0} \tau\left(\tilde{T}^{n}(M)_{\alpha}\right) \subseteq \oplus_{n \geq 0}\left(\tilde{T}^{n}(M)_{\alpha}+I\right)+\tilde{U}_{n-1}(M)\right) \subseteq(g r \tilde{U}(M))_{\alpha},
$$

as desired. Now for any $x \in M_{\alpha}, y \in M_{\beta}, z \in M_{\gamma}$, consider $\bar{x}=\iota(x), \bar{y}=\iota(y), \bar{z}=\iota(z)$ $\in(\operatorname{gr} \tilde{U}(M))_{1}$. Then, in $\operatorname{gr} \tilde{U}(M)$ there holds

$$
\begin{gathered}
\bar{x} \bar{y}-(-1)^{\alpha \beta} \bar{y} \bar{x}=\left(\iota(x) \iota(y)-(-1)^{\alpha \beta} \iota(y) \iota(x)\right)+\tilde{U}_{1}(M)=\iota([x, y])+\tilde{U}_{1}(M)=0 ; \\
(\bar{x} \bar{y}) \bar{z}-\bar{x}(\bar{y} \bar{z})=(\iota(x) \iota(y)) \iota(z)-\iota(x)(\iota(y) \iota(z))+\tilde{U}_{2}(M)=\iota(A(x, y, z))+\tilde{U}_{2}(M)=0 .
\end{gathered}
$$

This implies that $I^{*} \subseteq \operatorname{Ker} \tau$. Therefore, there is an epimorphism of $Z$-graded superalgebras $\tilde{\tau}: \tilde{T}(M) / I^{*} \rightarrow \operatorname{gr} \tilde{U}(M)$ such that $\tilde{\tau}\left(m+I^{*}\right)=\tau(m)=\iota(m)$, for all $m \in M$.

From the two previous lemmas, we know that the composite map $\tilde{\tau}^{-1}: V(M) \rightarrow \operatorname{gr} \tilde{U}(M)$ is an epimorphism of superalgebras satisfying $\tilde{\tau} \tilde{\pi}^{-1}(m)=\iota(m)$ for all $m \in M$. It is our aim, to prove that this epimorphism is in fact an isomorphism. For this we need to endow $V(M)$ with a convenient superalgebra structure.

Let $\left\{e_{r}, r \in \Delta\right\}$ be a basis of $M$ indexed by the totally ordered set $\Delta=\Delta_{0} \cup \Delta_{1}$ satisfying the following: $\left\{e_{r}: r \in \Delta_{\alpha}\right\}$ is a basis of $M_{\alpha}, \alpha=0,1$, and $r<s$ if $r \in \Delta_{0}, s \in \Delta_{1}$. It is well known that, in these conditions, $\left\{e_{r_{1}} e_{r_{2}}: r_{1} \leq r_{2}\right.$, and $r_{1}<r_{2}$ if $\left.r_{1}, r_{2} \in \Delta_{1}\right\}$ is a basis of $V^{2}(M)$ and $\left\{e_{r_{1}} e_{r_{2}} e_{r_{3}}: r_{1} \leq r_{2} \leq r_{3}\right.$, and $r_{p}<r_{p+1}$ if $\left.r_{p}, r_{p+1} \in \Delta_{1}\right\}$ is a basis of $V^{3}(M)$. In the supervector space $V(M)$ we define a new multiplication denoted by $*$ in the following way: if $a \in V^{i}(M), b \in V^{j}(M)$, then $a * b=a \otimes b$ if $i+j>3$; if $i+j \leq 3$ the multiplication is defined on the basis elements by the following identities (for simplicity we use $\bar{r}, \bar{s}, \bar{k}$ to denote the degrees of $e_{r}, e_{s}, e_{k}$, respectively):

$$
e_{r} * e_{s}= \begin{cases}e_{r} e_{s}, & \text { if } r \leq s \text { and } r \neq s \text { if } r \in \Delta_{1} \\ 1 / 2\left[e_{r}, e_{r}\right], & \text { if } r=s \in \Delta_{1} \\ (-1)^{\bar{r}} e_{s} e_{r}+\left[e_{r}, e_{s}\right], & \text { if } r>s\end{cases}
$$




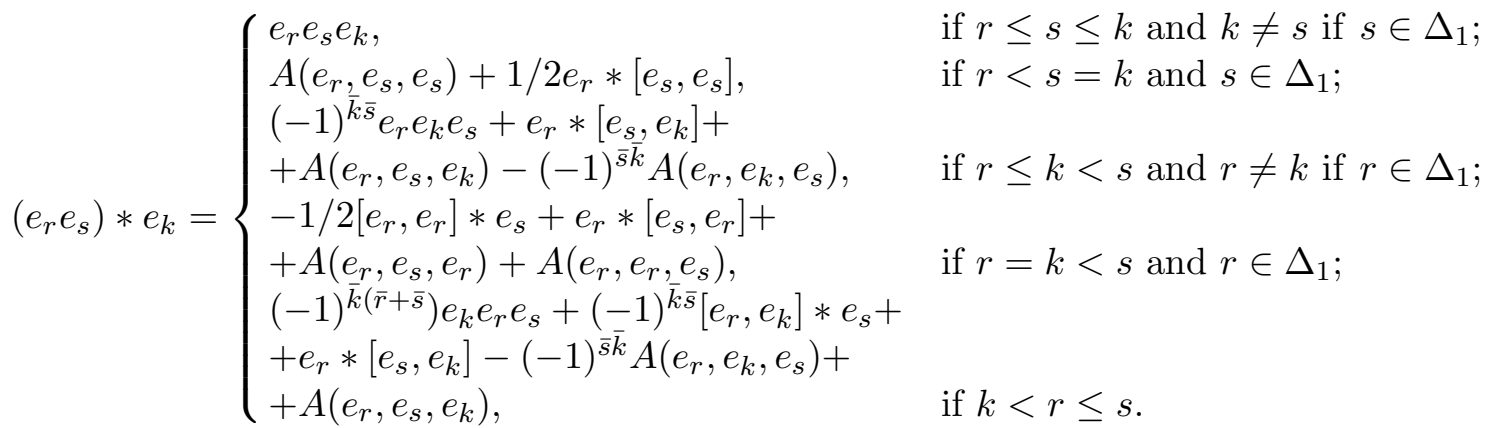

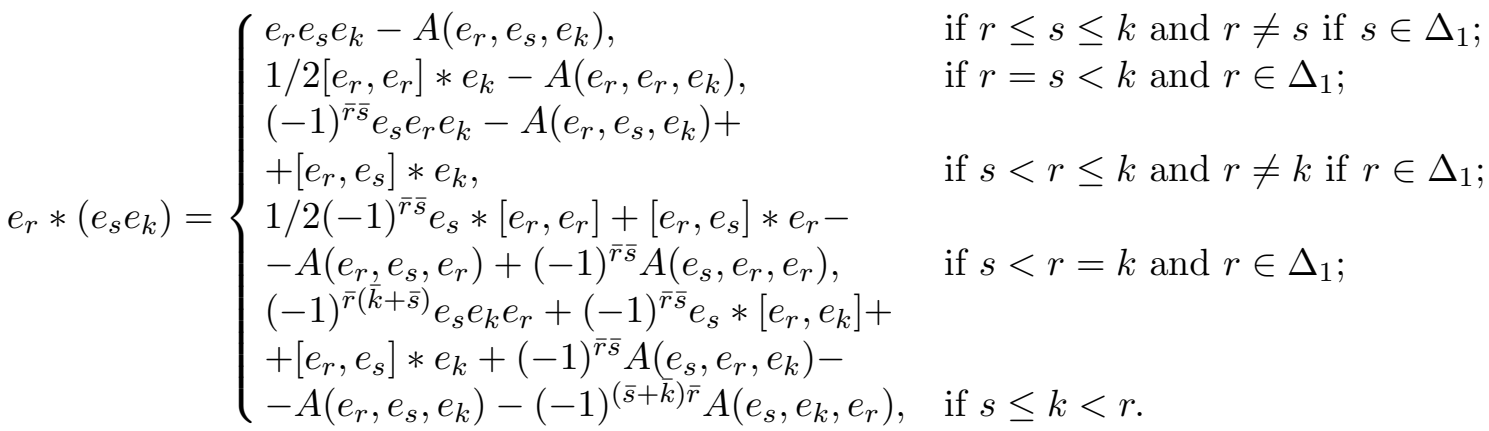

Note that if we consider a basis element $e_{p} e_{q}$ of $V^{2}(M)$ we always assume $p \leq q$ and $p \neq q$ if $p, q \in \Delta_{1}$.

With this multiplication $V(M)$ becomes a superalgebra that will be denoted by $\tilde{V}(M)$.

Lemma 3. There is an homomorphism of superalgebras $\hat{\epsilon}: \tilde{U}(M) \rightarrow \tilde{V}(M)$, of degree 0 , satisfying $\hat{\epsilon}(\iota(m))=m$, for all $m \in M$.

Proof: Denote the operations in the Akivis superalgebra $\tilde{V}(M)^{A}$ by

$$
<x, y>=x * y-(-1)^{\alpha \beta} y * x, \quad<x, y, z>=(x * y) * z-x *(y * z),
$$

for $x \in M_{\alpha}, y \in M_{\beta}, z \in M_{\gamma}$.

We start by proving that the inclusion map $\epsilon: M \rightarrow \tilde{V}(M)^{A}$ is an homomorphism of Akivis superalgebras. For this, it is enough to show that

$$
\left[e_{r}, e_{s}\right]=<e_{r}, e_{s}>\text { and } A\left(e_{r}, e_{s}, e_{k}\right)=<e_{r}, e_{s}, e_{k}>
$$

for the basis elements $e_{r}, e_{s}, e_{k}$ considered above. It is quite simple to see that the first of these two inequalities holds. For the second one, we have to consider several cases. Here we present only four of them, being the other cases similar.

1. $r=s=k \in \Delta_{1}$ :

$<e_{r}, e_{r}, e_{r}>=\left(e_{r} * e_{r}\right) * e_{r}-e_{r} *\left(e_{r} * e_{r}\right)=1 / 2\left(\left[e_{r}, e_{r}\right] * e_{r}-e_{r} *\left[e_{r}, e_{r}\right]\right)$.

As $\left[e_{r}, e_{r}\right] \in M_{0}$, we have $\left[e_{r}, e_{r}\right]=\sum_{t \in \Delta_{0}} \alpha_{t} e_{t}$ (sum with finite support), for scalars $\alpha_{t} \in K$. Therefore, as $t<r$ for any $t \in \Delta_{0}$, there holds

$$
\left[e_{r}, e_{r}\right] * e_{r}=\sum_{t \in \Delta_{0}} \alpha_{t} e_{t} * e_{r}=\sum_{t \in \Delta_{0}} \alpha_{t} e_{t} e_{r}
$$


In a similar way, we see that

$$
e_{r} *\left[e_{r}, e_{r}\right]=\sum_{t \in \Delta_{0}} \alpha_{t}\left(e_{t} e_{r}+\left[e_{r}, e_{t}\right]\right)=\sum_{t \in \Delta_{0}} \alpha_{t} e_{t} e_{r}+\left[e_{r},\left[e_{r}, e_{r}\right]\right]
$$

Hence $<e_{r}, e_{r}, e_{r}>=-1 / 2\left[e_{r},\left[e_{r}, e_{r}\right]\right]=1 / 2\left[\left[e_{r}, e_{r}\right], e_{r}\right]$. On the other hand, from the definition of Akivis superalgebra, we have that $S J\left(e_{r}, e_{r}, e_{r}\right)=3\left[\left[e_{r}, e_{r}\right], e_{r}\right]=$ $6 A\left(e_{r}, e_{r}, e_{r}\right)$. Thus $<e_{r}, e_{r}, e_{r}>=A\left(e_{r}, e_{r}, e_{r}\right)$.

2. $r \leq k<s$ and $r \neq k$ if $r \in \Delta_{1}$ :

$$
\begin{aligned}
& <e_{r}, e_{s}, e_{k}>=\left(e_{r} e_{s}\right) * e_{k}-e_{r} *\left((-1)^{\bar{s} \bar{k}} e_{k} e_{s}+\left[e_{s}, e_{k}\right]\right)=(-1)^{\bar{s} \bar{k}} e_{r} e_{k} e_{s}+A\left(e_{r}, e_{s}, e_{k}\right)- \\
& -(-1)^{\bar{s} \bar{k}} A\left(e_{r}, e_{k}, e_{s}\right)+e_{r} *\left[e_{s}, e_{k}\right]-e_{r} *\left[e_{s}, e_{k}\right]-(-1)^{\bar{s} \bar{k}} e_{r} e_{k} e_{s}+(-1)^{\bar{s} \bar{k}} A\left(e_{r}, e_{k}, e_{s}\right)= \\
& =A\left(e_{r}, e_{s}, e_{k}\right) .
\end{aligned}
$$

3. $r=k<s$ and $r \in \Delta_{1}$ :

$$
\begin{aligned}
& <e_{r}, e_{s}, e_{r}>=-1 / 2\left[e_{r}, e_{r}\right] * e_{s}+e_{r} *\left[e_{s}, e_{r}\right]+ \\
& +A\left(e_{r}, e_{s}, e_{r}\right)+A\left(e_{r}, e_{r}, e_{s}\right)+e_{r} *\left(e_{r} e_{s}\right)-e_{r} *\left[e_{s}, e_{r}\right]= \\
& =A\left(e_{r}, e_{s}, e_{r}\right)+A\left(e_{r}, e_{r}, e_{s}\right)-1 / 2\left[e_{r}, e_{r}\right] * e_{s}+1 / 2\left[e_{r}, e_{r}\right] * e_{s}-A\left(e_{r}, e_{r}, e_{s}\right)= \\
& =A\left(e_{r}, e_{s}, e_{r}\right) .
\end{aligned}
$$

4. $k<s<r$ :

$$
\begin{aligned}
& <e_{r}, e_{s}, e_{k}>=(-1)^{\bar{s} \bar{r}}\left(e_{s} e_{r}\right) * e_{k}+\left[e_{r}, e_{s}\right] * e_{k}-(-1)^{\bar{s} \bar{k}} e_{r} *\left(e_{k} e_{s}\right)-e_{r} *\left[e_{s}, e_{k}\right]= \\
& =(-1)^{(\bar{s}+\bar{k}) \bar{r}}\left[e_{s}, e_{k}\right] * e_{r}+(-1)^{\bar{s} \bar{r}} e_{s} *\left[e_{r}, e_{k}\right]+\left[e_{r}, e_{s}\right] * e_{k}-e_{r} *\left[e_{s}, e_{k}\right]- \\
& -(-1)^{(\bar{s}+\bar{r}) \bar{k}} e_{k} *\left[e_{r}, e_{s}\right]-(-1)^{\bar{s} \bar{k}}\left[e_{r}, e_{k}\right] * e_{s}+(-1)^{\bar{s} \bar{r}} A\left(e_{s}, e_{r}, e_{k}\right)- \\
& -(-1)^{(\bar{s}+\bar{k}) \bar{r}} A\left(e_{s}, e_{k}, e_{r}\right)+(-1)^{\bar{s} \bar{k}+(\bar{s}+\bar{k}) \bar{r}} A\left(e_{k}, e_{s}, e_{r}\right)-(-1)^{(\bar{s}+\bar{r}) \bar{k}} A\left(e_{k}, e_{r}, e_{s}\right)+ \\
& +(-1)^{\bar{s} \bar{k}} A\left(e_{r}, e_{k}, e_{s}\right)=(\text { since }[x, y]=<x, y>\text { all } x, y \in M) \\
& =S J\left(e_{r}, e_{s}, e_{k}\right)-\left((-1)^{\bar{s} \bar{r}} A\left(e_{s}, e_{r}, e_{k}\right)-(-1)^{(\bar{s}+\bar{k}) \bar{r}} A\left(e_{s}, e_{k}, e_{r}\right)+\right. \\
& \left.+(-1)^{\bar{s} \bar{k}+(\bar{s}+\bar{k}) \bar{r}} A\left(e_{k}, e_{s}, e_{r}\right)-(-1)^{(\bar{s}+\bar{r}) \bar{k}} A\left(e_{k}, e_{r}, e_{s}\right)+(-1)^{\bar{s} \bar{k}} A\left(e_{r}, e_{k}, e_{s}\right)\right)= \\
& =A\left(e_{r}, e_{s}, e_{k}\right) \quad \text { by the definition of Akivis superalgebra). }
\end{aligned}
$$

As $\epsilon$ is an Akivis homomorphism, from the definition of enveloping superalgebra, there is an homomorphism of superalgebras of degree $0, \hat{\epsilon}: \tilde{U}(M) \rightarrow \tilde{V}(M)$ satisfying $\hat{\epsilon}(\iota(m))=m$, all $m \in M$.

Theorem 1. The $Z$-graded superalgebras $V(M)$ and $\operatorname{gr} \tilde{U}(M)$ are isomorphic.

Proof: The superalgebra $\tilde{V}(M)$ has a natural filtration defined by the sequence of subspaces $\tilde{V}_{n}(M)=\oplus_{i=0}^{n} V^{i}(M)$. So we may consider the associated $Z$-graded superalgebra $g r \tilde{V}(M)$. ( As usual we identify $(\operatorname{gr} \tilde{V}(M))_{1}$ with $M$ ). Since the map $\hat{\epsilon}$, considered in the previous lemma, is an homomorphism of superalgebras, we have $\hat{\epsilon}\left(\tilde{U}_{n}(M)\right) \subseteq \tilde{V}_{n}(M)$. So, we may define $\tilde{\epsilon}: \operatorname{gr} \tilde{U}(M) \rightarrow \operatorname{gr} \tilde{V}(M)$ by $\tilde{\epsilon}\left(a_{i}+\tilde{U}_{i-1}(M)\right)=\hat{\epsilon}\left(a_{i}\right)+\tilde{V}_{i-1}(M)$. This map is an homomorphism of $Z$-graded superalgebras of degree 0 and satisfies $\tilde{\epsilon}(\iota(m))=m$, all $m \in M$. We now return to the algebra $V(M)$. For $n \geq 1$, the map $\mu_{n}: V^{n}(M) \rightarrow(\operatorname{gr} \tilde{V}(M))_{n}$ defined by $\mu_{n}(v)=v+\tilde{V}_{n-1}(M)$ is an isomorphism of vector spaces. So taking $\mu_{0}=I d_{K}$, $\mu=\oplus_{n \geq 0} \mu_{n}: V(M) \rightarrow g r \tilde{V}(M)$ is an isomorphism of $Z$-graded vector spaces. Looking at the formulas which define the multiplication in $\tilde{V}(M)$, it is easy to see that this is in fact an 
isomorphism of $Z$-graded superalgebras. The composite homomorphisms $\mu^{-1} \tilde{\epsilon}: \operatorname{gr} \tilde{U}(M) \rightarrow$ $g r \tilde{V}(M) \rightarrow V(M)$ and $\tilde{\tau} \tilde{\pi}^{-1}: V(M) \rightarrow \tilde{T}(M) / I^{*} \rightarrow g r \tilde{U}(M)$ (recall the preceeding lemmas) are inverse of each other. So the result follows.

The following results are immediate consequence of this theorem.

Corollary 1. The canonical map $\iota: M \rightarrow \tilde{U}(M)$ is injective.

Corollary 2. Any Akivis superalgebra, defined over a field of characteristic different from 2 and 3, is special.

\section{BIBLIOGRAPHY}

[1] Akivis M. A., "Local algebras of a multidimensional three web" (Russian), Sibirsk. Mat. Zh. 17 (1), (1976) 5-11;

[2] Albuquerque H. and Shahn Majid, "Quasialgebra structure of the octonions", Journal of Algebra 220, (1999) 188-224;

[3] Albuquerque H., A. Elduque and José Pérez-Izquierdo, " $Z_{2^{-}}$quasialgebras", Com. in Algebra 30 (5), (2002) 2161-2174;

[4] Pérez-Izquierdo J., "Algebras, hyperalgebras, nonassociative bialgebras and loops", Advances in Mathematics 208 (2007) 834-876;

[5] Pérez-Izquierdo J. and Ivan Shestakov, "An envelope for Malcev algebras", J. of Algebra 272 (1) (2005) 379-393;

[6] Shestakov I., "Every Akivis Algebra is Linear", Geometriae Dedicata 77 (2) (1999) 215-223;

[7] Shestakov I. and U. U. Umirbaev, "Free Akivis Algebras, primitive elements and hyperalgebras", J. Algebra 250(2) (2002) 533-548.

[8] Sheunert M., " The theory of Lie superalgebras", LNM 716 Springer Verlag, Berlin 1979. 